\title{
GENERAL QUASILINEAR PROBLEMS INVOLVING $p(x)$-LAPLACIAN WITH ROBIN BOUNDARY CONDITION
}

\author{
Hassan Belaouidel ${ }^{1}$, Anass Ourraoui ${ }^{2}$, Najib Tsouli $^{3}$ \\ Department of Mathematics and Computer Science, \\ Faculty of Sciences, University Mohamed I, \\ Mohammed V av., P.O. Box 524, Oujda 60000, Morocco \\ ${ }^{1}$ belaouidelhassan@hotmail.fr, $\quad 2$ a.ourraoui@gmail.com, $\quad{ }^{3}$ tsouli@hotmail.fr
}

\begin{abstract}
This paper deals with the existence and multiplicity of solutions for a class of quasilinear problems involving $p(x)$-Laplace type equation, namely

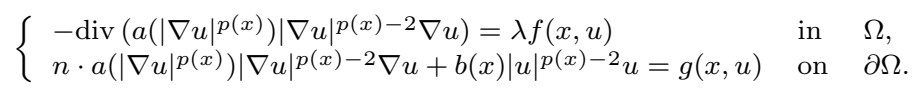

Our technical approach is based on variational methods, especially, the mountain pass theorem and the symmetric mountain pass theorem.
\end{abstract}

Keywords: $p(x)$-Laplacian, Mountain pass theorem, Multiple solutions, Critical point theory.

\section{Introduction}

In this paper we study the nonlinear elliptic boundary value problem with Robin conditions

$$
\begin{cases}-\operatorname{div}\left(a\left(|\nabla u|^{p(x)}\right)|\nabla u|^{p(x)-2} \nabla u\right)=\lambda f(x, u) & \text { in } \Omega, \\ n \cdot a\left(|\nabla u|^{p(x)}\right)|\nabla u|^{p(x)-2} \nabla u+b(x)|u|^{p(x)-2} u=g(x, u) & \text { on } \quad \partial \Omega,\end{cases}
$$

where $\Omega$ is an open bounded subset of $\mathbb{R}^{N}(N \geq 2)$, with smooth boundary, $n$ is the outer unit normal vector on $\partial \Omega, b$ is a positive continuous function defined on $\mathbb{R}^{N}, p \in C_{+}(\bar{\Omega})$ with

$$
1<p^{-}:=\inf _{\bar{\Omega}} p(x) \leq p^{+}:=\sup _{\bar{\Omega}} p(x)<N
$$

and $p(x)<p^{*}(x)$ where

$$
p^{*}(x)=\left\{\begin{array}{lll}
\frac{N p(x)}{N-p(x)} & \text { if } & p(x)<N \\
+\infty & \text { if } & p(x) \geq N
\end{array}\right.
$$

for any $x \in \bar{\Omega}$. It is clear that the equation in question is elliptic since it describes phenomena that do not change from moment to moment, and that the operator

$$
L u=-\operatorname{div}\left(a\left(|\nabla u|^{p(x)}\right)|\nabla u|^{p(x)-2} \nabla u\right)
$$

is an elliptic operator in divergence form.

Recently, the study of differential equations and variational problems involving $p(x)$-growth conditions have been extensively investigated and received much attention because they can be presented as models for many physical phenomena which arouse in the study of elastic mechanics [32], electro-rheological fluid dynamics [27] and image processing [6], electrical resistivity and 
polycrystal plasticity [3, 4] and continuum mechanics [2] etc, for an overview of this subject, and for more details we refer readers to [11] and [5, 10] and the references therein. The existence of nontrivial solutions to nonlinear elliptic boundary value problems has been extensively studied by many researchers $[1,7,14,15,18,21,23,24]$ and references therein.

It is known that the extension $p(x)$-Laplace operator possesses more complicated structure than the $p$-Laplacian. For example, it is inhomogeneous and usually it does not have the so-called first eigenvalue, since the infimum of its spectrum is zero.

However, to understand the role of the variable exponent, well, although most of the materials can be accurately modeled with the help of the classical Lebesgue and Sobolev spaces $L^{p}$ and $W^{1, p}$, where $p$ is a fixed constant, there are some nonhomogeneous materials, for which this is not adequate, e.g. the rheological fluids mentioned above, which are characterized by their ability to drastically change their mechanical properties under the influence of an exterior electromagnetic field. Thus it is necessary for the exponent $p$ to be nonstandard, therefore, the spaces with variable exponents are required. As an introduction and a history coverage to the subject of variable exponent problems, we advice the reader to see the monograph [12] and the articles [16, 20].

Note that, the $p(x)$-Laplace operator in (1.1) is a special case of the divergence form operator $-\operatorname{div}\left(a\left(|\nabla u|^{p(x)}\right)|\nabla u|^{p(x)-2} \nabla u\right)$ which appears in many nonlinear diffusion problems, in particular in the mathematical modeling of non-Newtonian fluids. When

$$
a(t)=1+\frac{t}{\sqrt{1+t^{2}}}
$$

we have the generalized Capillary operator (which is essential in applied fields like industrial, biomedical and pharmaceutical) initiated by W. Ni and J. Serrin [22].

Inspired by the works in [25] and [19], we study the existence and multiplicity of nontrivial solutions the problem (1.1), via the mountain pass theorem and the Rabinowitz's symmetric mountain pass theorem [26].

We assume the following conditions:

$\left(\mathbf{A}_{0}\right)$ The function $a: \mathbb{R}^{+} \rightarrow \mathbb{R}$ is continuous and the mapping $\Theta: \mathbb{R}^{N} \rightarrow \mathbb{R}$, given by $\Theta(\xi)=A\left(|\xi|^{p(x)}\right)$ is strictly convex, where $A$ is the primitive of $a$, that is

$$
A(t)=\int_{0}^{t} a(s) d s
$$

$\left(\mathbf{A}_{1}\right)$ There exist two constants $0<L<K$ such that $L \leq a(t) \leq K$ for all $t \geq 0$.

We assume that $f, g: \Omega \times \mathbb{R} \rightarrow \mathbb{R}$ are of Carathéodory functions, $f(x, \cdot)=g(x, \cdot)=0$ and satisfy:

$\left(\mathbf{F}_{0}\right)$ for all $(x, t) \in \Omega \times \mathbb{R}|f(x, t)| \leq f_{1}(x)|t|^{r(x)-1}$, such that

$$
1 \leq r^{-}:=\inf _{\bar{\Omega}} r(x) \leq r^{+}:=\sup _{\bar{\Omega}} r(x)<p^{-}:=\inf _{\bar{\Omega}} p(x) \leq p^{+}:=\sup _{\bar{\Omega}} p(x),
$$

where $f_{1}$ is nonnegative, measurable function and $f_{1} \in L^{\frac{p(x)}{p(x)-r(x)}}(\Omega)$;

$\left(\mathbf{F}_{1}\right)$ for all $(x, t) \in \Omega \times \mathbb{R}|f(x, t)| \geq f_{2}(x)|t|^{\alpha(x)-1}$,

$$
1 \leq \alpha^{-}: \inf _{\bar{\Omega}} \alpha(x) \leq \alpha^{+}:=\sup _{\bar{\Omega}} \alpha(x)<r^{-},
$$

where $f_{2}>0$ in some nonempty open set $O \subset \Omega$; 
$\left(\mathbf{G}_{0}\right)$ for all $(x, t) \in \partial \Omega \times \mathbb{R},|g(x, t)| \leq g_{1}(x)|t|^{q(x)-1}$,

$$
1 \leq p^{+}<q^{-}:=\inf _{\bar{\Omega}} q(x) \leq q^{+}:=\sup _{\bar{\Omega}} q(x), \quad q(x)<p^{\partial}(x),
$$

where

$$
p^{\partial}(x)=(p(x))^{\partial}=\left\{\begin{array}{lll}
\frac{(N-1) p(x)}{N-p(x)} & \text { if } & p(x)<N \\
+\infty & \text { if } & p(x) \geq N
\end{array}\right.
$$

and there exists a positive constants $C_{g}$ such that $0 \leq g_{1} \leq C_{g}$;

$\left(\mathbf{G}_{1}\right)$ for all $(x, t) \in \partial \Omega \times \mathbb{R} \lim _{t \rightarrow 0} \frac{g(x, t) t}{|t|^{p^{+}-1}}=0$.

$\left(\mathbf{G}_{2}\right)$ there exists $\mu>p^{+}$such that $\mu G(x, t) \leq g(x, t) t$ for all $(x, t) \in \partial \Omega \times \mathbb{R}$, where

$$
G(x, t)=\int_{0}^{t} g(x, s) d s .
$$

The main result of this paper is as follow.

Theorem 1. Assume that $\left(\mathbf{A}_{0}\right)-\left(\mathbf{A}_{1}\right),\left(\mathbf{F}_{0}\right)-\left(\mathbf{F}_{1}\right)$ and $\left(\mathbf{G}_{0}\right)-\left(\mathbf{G}_{2}\right)$ hold. Then there exists $\lambda^{*}>0$ such that for every $\left.\lambda \in\right] 0, \lambda^{*}[$, the problem (1.1) admits at least one nontrivial solution. In addition, if we assume the following conditions:

$\left(\mathbf{G}_{3}\right)$ there is a nonempty open set $U \subset \partial \Omega$ with $G(x, t)>0$ for all $(x, t) \in U \times \mathbb{R}^{+}$,

$\left(\mathbf{G}_{4}\right)$ the functions $f$ and $g$ are even,

then the problem (1.1) has infinitely many solutions for every $\lambda>0$.

The remainder of this paper is organized as follows, in Section 2 we introduce some technical results and required hypotheses in order to solve our problem, in Section 3 we state some and prove the main results of this work.

\section{Preliminaries}

In the sequel, let $p(x) \in C_{+}(\bar{\Omega})$, where

$$
C_{+}(\bar{\Omega})=\{h: h \in C(\bar{\Omega}), h(x)>1 \text { for any } x \in \bar{\Omega}\} .
$$

The variable exponent Lebesgue space is defined by

$$
L^{p(x)}(\Omega)=\left\{u: \Omega \rightarrow \mathbb{R} \text { measurable and } \int_{\Omega}|u(x)|^{p(x)} d x<+\infty\right\}
$$

furnished with the Luxemburg norm

$$
|u|_{L^{p(x)}(\Omega)}=|u|_{p(x)}=\inf \left\{\sigma>0: \int_{\Omega}\left|\frac{u(x)}{\sigma}\right|^{p(x)} d x \leq 1\right\} .
$$

Remark 1. Variable exponent Lebesgue spaces resemble to classical Lebesgue spaces in many respects, they are separable Banach spaces and the Hölder inequality holds. The inclusions between Lebesgue spaces are also naturally generalized, that is, if $0<\operatorname{mes}(\Omega)<\infty$ and $p, q$ are 
variable exponents such that $p(x)<q(x)$ a. e. in $\Omega$, then there exists a continuous embedding $L^{q(x)}(\Omega) \hookrightarrow L^{p(x)}(\Omega)$.

The variable exponent Sobolev space is defined by

$$
W^{1, p(x)}(\Omega)=\left\{u \in L^{p(x)}(\Omega):|\nabla u| \in L^{p(x)}(\Omega)\right\}
$$

equipped with the norm

$$
\|u\|_{W^{1, p(x)}(\Omega)}=|u|_{L^{p(x)}(\Omega)}+|\nabla u|_{L^{p(x)}(\Omega)} .
$$

Proposition $1[16,17]$. The spaces $L^{p(x)}(\Omega)$ and $W^{1, p(x)}(\Omega)$ are separable, uniformly convex, reflexive Banach spaces. The conjugate space of $L^{p(x)}(\Omega)$ is $L^{q(x)}(\Omega)$, where $q(x)$ is the conjugate function of $p(x)$, i.e.,

$$
\frac{1}{p(x)}+\frac{1}{q(x)}=1
$$

for all $x \in \Omega$. For $u \in L^{p(x)}(\Omega)$ and $v \in L^{q(x)}(\Omega)$ we have

$$
\left|\int_{\Omega} u(x) v(x) d x\right| \leq\left(\frac{1}{p^{-}}+\frac{1}{q^{-}}\right)|u|_{p(x)}|v|_{q(x)} .
$$

Moreover, if $h_{1}, h_{2}, h_{3}: \bar{\Omega} \rightarrow(1, \infty)$ are Lipschitz continuous functions such that

$$
\frac{1}{h_{1}}+\frac{1}{h_{2}}+\frac{1}{h_{3}}=1
$$

then for any $u \in L^{h_{1}(x)}(\Omega), v \in L^{h_{2}(x)}(\Omega), w \in L^{h_{3}(x)}(\Omega)$, the following inequality holds (see [15, Proposition 2.5])

$$
\int_{\Omega}|u v w| d x \leq\left(\frac{1}{h_{1}^{-}}+\frac{1}{h_{2}^{-}}+\frac{1}{h_{3}^{-}}\right)|u|_{h_{1}(x)}|v|_{h_{2}(x)}|w|_{h_{3}(x)} .
$$

Proposition 2 [13]. Let $p(x)$ and $q(x)$ be measurable functions such that $p(x) \in L^{\infty}(\Omega)$ and $1 \leq p(x) q(x) \leq \infty$, for a.e. $x \in \Omega$. Let $u \in L^{q(x)}(\Omega), u \neq 0$. Then

$$
\begin{gathered}
|u|_{p(x) q(x)} \leq 1 \Rightarrow|u|_{p(x) q(x)}^{p^{+}} \leq\left.\left.|| u\right|^{p(x)}\right|_{q(x)} \leq|u|_{p(x) q(x)}^{p^{-}} \\
|u|_{p(x) q(x)} \geq 1 \Rightarrow|u|_{p(x) q(x)}^{p^{-}} \leq\left.\left.|| u\right|^{p(x)}\right|_{q(x)} \leq|u|_{p(x) q(x)}^{p^{+}}
\end{gathered}
$$

In particular if $p(x)=p$ is a constant, then

$$
\left.\left.|| u\right|^{p}\right|_{q(x)}=|u|_{p q(x)}^{p} .
$$

Proposition $3[16,17]$. Assume that the boundary of $\Omega$ possesses the cone property and $p, r \in C_{+}(\bar{\Omega})$ such that $r(x) \leq p^{*}(x)\left(r(x)<p^{*}(x)\right)$ for all $x \in \bar{\Omega}$, then there is a continuous (compact) embedding

$$
W^{1, p(x)}(\Omega) \hookrightarrow L^{r(x)}(\Omega),
$$

Proposition 4 [9]. For $p \in C_{+}(\bar{\Omega})$ and such $r \in C_{+}(\partial \Omega)$ that $r(x) \leq p^{\partial}(x)\left(r(x)<p^{\partial}(x)\right)$ for all $x \in \bar{\Omega}$, there is a continuous (compact) embedding

$$
W^{1, p(x)}(\Omega) \hookrightarrow L^{r(x)}(\partial \Omega) .
$$


Proposition 5. [8, Theorem 2.1] For any $u \in W^{1, p(x)}(\Omega)$, let

$$
\|u\|_{\partial}:=|u|_{L^{p(x)}(\partial \Omega)}+|\nabla u|_{L^{p(x)}(\Omega)} .
$$

Then $\|u\|_{\partial}$ is a norm on $W^{1, p(x)}(\Omega)$ which is equivalent to

$$
\|u\|_{W^{1, p(x)}(\Omega)}=|u|_{L^{p(x)}(\Omega)}+|\nabla u|_{L^{p(x)}(\Omega)} .
$$

Now, for any $u \in X:=W^{1, p(x)}(\Omega)$ define

$$
\|u\|:=\inf \left\{\sigma>0: \int_{\Omega}\left|\frac{\nabla u(x)}{\sigma}\right|^{p(x)} d x+\int_{\partial \Omega} b(x)\left|\frac{u(x)}{\sigma}\right|^{p(x)} d \sigma_{x} \leq 1\right\},
$$

where $b \in L^{\infty}(\Omega)$ and $d \sigma_{x}$ is the measure on the boundary $\partial \Omega$. Then by Proposition $5,\|\cdot\|$ is also a norm on $W^{1, p(x)}(\Omega)$ which is equivalent to $\|\cdot\|_{W^{1, p(x)}(\Omega)}$ and $\|\cdot\|_{\partial}$, the proof of this statement can be found in $[8$, p. 551]. Now, we introduce the modular $\rho: X \rightarrow \mathbb{R}$ defined by

$$
\rho(u)=\int_{\Omega}|\nabla u|^{p(x)} d x+\int_{\partial \Omega} b(x)|u(x)|^{p(x)} d \sigma_{x}
$$

for all $u \in X$. Here, we give some relations between the norm $\|\cdot\|$ and the modular $\rho$.

Proposition 6 [16]. For $u \in X$ we have

(i) $\|u\|<1(=1 ;>1) \Leftrightarrow \rho(u)<1(=1 ;>1)$;

(ii) If $\|u\|<1 \Rightarrow\|u\|^{p^{+}} \leq \rho(u) \leq\|u\|^{p^{-}}$;

(iii) If $\|u\|>1 \Rightarrow\|u\|^{p^{-}} \leq \rho(u) \leq\|u\|^{p^{+}}$.

Proposition 7 [29]. Suppose that $f: \Omega \times \mathbb{R} \rightarrow \mathbb{R}$ is a Carathéodory function and satisfies the growth condition

$$
|f(x, t)| \leq c|t|^{\alpha(x) / \beta(x)}+h(x), \quad \text { for every } x \in \Omega, t \in \mathbb{R},
$$

where $\alpha, \beta \in C_{+}(\bar{\Omega}), c \geq 0$ is constant and $h \in L^{\beta(x)}(\Omega)$. Then $N_{f}\left(L^{\alpha(x)}(\Omega)\right) \subseteq L^{\beta(x)}(\Omega)$, where $N_{f}(u)(x)=f\left(x, u(x)\right.$. Moreover, $N_{f}$ is continuous from $L^{\alpha(x)}(\Omega)$ into $L^{\beta(x)}(\Omega)$ and maps bounded set into bounded set.

As a consequence of Proposition 7, the Carathéodory function $f$ defines an operator $N_{f}$ which is called the Nemytskii operator.

Definition 1. We say that $u \in X$ is weak solution of (1.1) if

$$
\int_{\Omega} a\left(|\nabla u|^{p(x)}\right)|\nabla u|^{p(x)-2} \nabla u \nabla v d x+\int_{\partial \Omega} b(x)|u|^{p(x)-2} u v d \sigma_{x}=\lambda \int_{\Omega} f(x, u) v d x+\int_{\partial \Omega} g(x, u) v d \sigma_{x}
$$

for all $v \in X$.

Now we introduce the Euler-Lagrange functional $I_{\lambda}: X \longrightarrow \mathbb{R}$ associated with problem (1.1) defined by

$$
I_{\lambda}(u)=\int_{\Omega} \frac{1}{p(x)} A\left(|\nabla u|^{p(x)}\right) d x+\int_{\partial \Omega} \frac{1}{p(x)} b(x)|u|^{p(x)} d \sigma_{x}-\lambda \int_{\Omega} F(x, u) d x-\int_{\partial \Omega} G(x, u) d \sigma_{x},
$$

where

$$
F(x, t):=\int_{0}^{t} f(x, s) d s .
$$

Furthermore, the (weak) solutions of (1.1) are precisely the critical points of the functional $I_{\lambda}$. 
Lemma 1 [31]. Let

$$
L(u):=\int_{\Omega} \frac{1}{p(x)} A\left(|\nabla u|^{p(x)}\right) d x+\int_{\partial \Omega} \frac{1}{p(x)} b(x)|u|^{p(x)} d \sigma_{x} .
$$

Then the mapping $L: X \rightarrow X^{*}$ is a strictly monotone, continuous bounded homeomorphism and is of type $\left(S_{+}\right)$, namely assumptions $u_{n} \rightarrow u$ and $\lim \sup L\left(u_{n}\right)\left(u_{n}-u\right) \leq 0$, imply $u_{n} \rightarrow u$.

By Proposition 7, we can see that the functional $I_{\lambda}$ is well defined on $X$ and $I_{\lambda} \in C^{1}(X, \mathbb{R})$ with its Fréchet derivative is giving by

$$
\begin{gathered}
I_{\lambda}^{\prime}(u) \cdot v=\int_{\Omega} a\left(|\nabla u|^{p(x)}\right)|\nabla u|^{p(x)-2} \nabla u \nabla v d x+\int_{\partial \Omega} b(x)|u|^{p(x)-2} u v d \sigma_{x} \\
-\lambda \int_{\Omega} f(x, u) v d x-\int_{\partial \Omega} g(x, u) v d \sigma_{x}
\end{gathered}
$$

for all $u, v \in X$.

Let $X$ be a real Banach space and let be a functional $I \in \mathcal{C}^{1}(X, \mathbb{R})$. We say that $I$ satisfies the Palais-Smale condition on $X\left((P S)\right.$-condition, for short) if any sequence $\left(u_{n}\right) \subset X$ with $\left(I\left(u_{n}\right)\right)$ bounded and $I^{\prime}\left(u_{n}\right) \rightarrow 0$ as $n \rightarrow \infty$, possesses a convergent subsequence. By $(P S)$-sequence for $I$ we understand a sequence $\left(u_{n}\right) \subset X$ which satisfies the conditions: $\left(I\left(u_{n}\right)\right)$ is bounded and $I^{\prime}\left(u_{n}\right) \rightarrow 0$ as $n \rightarrow \infty$.

The main tools used in proving Theorem 1 are the well known mountain pass theorem and its the symmetric mountain pass theorem.

Theorem 2 [26, Theorem 2.2]. Let $X$ be a real Banach space and let I belong to $\mathcal{C}^{1}(X, \mathbb{R})$ satisfying the $(P S)$-condition. Suppose that $I(0)=0$ and that the following conditions hold:

$\left(\mathbf{I}_{1}\right)$ there exist $\rho>0$ and $\varrho>0$ such that $I(u) \geq \varrho$ for $\|u\|=\rho$;

$\left(\mathbf{I}_{2}\right)$ there exists $e \in X$ with $\|e\|>\rho$ such that $I(e) \leq 0$.

Let

$$
\Gamma=\{\gamma \in \mathcal{C}([0,1] ; X): \gamma(0)=0, \gamma(1)=e\}, \quad c=\inf _{\gamma \in \Gamma} \max _{0 \leq t \leq 1} I(\gamma(t)),
$$

then, $c$ is a critical value of $I$.

Theorem 3 [28, Theorem 2.1]. Let $X$ be a real Banach space and let I belong to $\mathcal{C}^{1}(X, \mathbb{R})$ be even, satisfies $(P S)$-condition and $I(0)=0$. If $X=Y \oplus Z$ with $\operatorname{dim} Y<\infty$, and I satisfies

$\left(\mathbf{I}_{1}\right)$ there are constants $\rho,>0$ such that $I / \partial B_{\rho} \cap Z \geq 0$

$\left(\mathbf{I}_{2}\right)$ there a finite dimensional subspace $W \subset X$, with $\operatorname{dim} Y<\operatorname{dim} W<\infty$ and there is $M>0$ such that $\max _{u \in W} I(u)<M$

$\left(\mathbf{I}_{3}\right)$ considering $M>0$ given by $\left(\mathbf{I}_{2}\right)$, I satisfies $(P S)_{c}$ for $0 \leq c \leq M$.

Then I possesses at least $\operatorname{dim} W-\operatorname{dim} Y$ pairs of nontrivial critical points. 


\section{Proof of Theorem 1}

To prove Theorem 1 we recall some lemmas presented below.

Lemma 2. Assume that $\left(\mathbf{A}_{1}\right),\left(\mathbf{F}_{0}\right)$ and $\left(\mathbf{G}_{2}\right)$ hold. Then the functional $I_{\lambda}$ satisfies the PalaisSmale condition on $X((P S)$-condition, for short) at any level $d$.

P r o o f. Let $d \in \mathbb{R}$ and let $\left(u_{n}\right) \subset X$ be $(P S)$ sequence for $I_{\lambda}$, then

$$
I_{\lambda}\left(u_{n}\right) \rightarrow d \quad \text { and } \quad I_{\lambda}^{\prime}\left(u_{n}\right) \rightarrow 0 \quad \text { as } \quad n \rightarrow \infty .
$$

First, we prove that sequence $\left(u_{n}\right)$ is bounded in $X$. Suppose $\left(u_{n}\right)$ unbounded, we may assume $\left\|u_{n}\right\| \rightarrow+\infty$ as $n \rightarrow \infty$.

By $(2),\left(\mathbf{A}_{1}\right),\left(\mathbf{F}_{0}\right)$ and Proposition 6 we have

$$
\begin{gathered}
I_{\lambda}\left(u_{n}\right)=\int_{\Omega} \frac{1}{p(x)} A\left(\left|\nabla u_{n}\right|^{p(x)}\right) d x+\int_{\partial \Omega} \frac{1}{p(x)} b(x)\left|u_{n}\right|^{p(x)} d \sigma_{x} \\
-\lambda \int_{\Omega} F\left(x, u_{n}\right) d x-\int_{\partial \Omega} G\left(x, u_{n}\right) d \sigma_{x} \\
\geq \frac{L}{p^{+}} \int_{\Omega}\left|\nabla u_{n}\right|^{p(x)} d x+\int_{\partial \Omega} \frac{1}{p^{+}} b(x)\left|u_{n}\right|^{p(x)} d \sigma_{x}-\frac{\lambda}{r^{+}} \int_{\Omega} f_{1}(x)\left|u_{n}\right|^{r(x)} d x-\int_{\partial \Omega} G\left(x, u_{n}\right) d \sigma_{x} \\
\geq \frac{\min (L, 1)}{p^{+}}\left\|u_{n}\right\|^{p^{-}}-\frac{\lambda}{r^{+}} \int_{\Omega} f_{1}(x)\left|u_{n}\right|^{r(x)} d x-\int_{\partial \Omega} G\left(x, u_{n}\right) d \sigma_{x} .
\end{gathered}
$$

From (3.2), $\left(\mathbf{F}_{0}\right)$ and Proposition 6 we obtain

$$
\begin{gathered}
\frac{1}{\mu} I_{\lambda}^{\prime}\left(u_{n}\right) \cdot u_{n}=\frac{1}{\mu} \int_{\Omega} a\left(\left|\nabla u_{n}\right|^{p(x)}\right)\left|\nabla u_{n}\right|^{p(x)} d x+\frac{1}{\mu} \int_{\partial \Omega} b(x)\left|u_{n}\right|^{p(x)} d \sigma_{x} \\
\quad-\frac{\lambda}{\mu} \int_{\Omega} f\left(x, u_{n}\right) u_{n} d x-\frac{1}{\mu} \int_{\partial \Omega} g\left(x, u_{n}\right) u_{n} d \sigma_{x} \\
\geq \frac{\min (L, 1)}{\mu}\left\|u_{n}\right\|^{p^{-}}-\frac{\lambda}{\mu} \int_{\Omega} f_{1}(x)\left|u_{n}\right|^{r(x)} d x-\frac{1}{\mu} \int_{\partial \Omega} g\left(x, u_{n}\right) u_{n} d \sigma_{x} .
\end{gathered}
$$

Meanwhile, according to $\left(\mathbf{F}_{0}\right)$, Proposition 4 and Proposition 2 it yields

$$
\begin{gathered}
\int_{\Omega} f_{1}(x)\left|u_{n}\right|^{r(x)} d x \leq \int_{\Omega}\left|f_{1}(x)\right|\left|u_{n}\right|^{r(x)} d x \leq\left.\left.\left|f_{1}\right|_{L^{\frac{p(x)}{p(x)-r(x)}(\Omega)}}|| u_{n}\right|^{r(x)}\right|_{\frac{p(x)}{r(x)}} \\
\leq\left|f_{1}\right|_{L^{\frac{p(x)}{p(x)-r(x)}(\Omega)}} \max \left(\left|u_{n}\right|_{p(x)}^{r^{-}},\left|u_{n}\right|_{p(x)}^{r^{+}}\right) \leq\left|f_{1}\right|_{L^{\frac{p(x)}{p(x)-r(x)}(\Omega)}} \max \left(C_{r^{-}}\left\|u_{n}\right\|^{r^{-}}, C_{r^{+}}\left\|u_{n}\right\|^{r^{+}}\right),
\end{gathered}
$$

where $C_{r^{-}}$and $C_{r^{+}}$are constants of compact embedding $X \hookrightarrow L^{p(x)}(\Omega)$. Using (3.1), (3.2), (3.3), $(3.4)$ and $\left(G_{2}\right)$ we obtain

$$
\begin{gathered}
d+1+\left\|u_{n}\right\| \geq I_{\lambda}\left(u_{n}\right)-\frac{1}{\mu} I_{\lambda}^{\prime}\left(u_{n}\right) \cdot u_{n} \geq \frac{\min (L, 1)}{p^{+}}\left\|u_{n}\right\|^{p^{-}}-\frac{\lambda}{r^{+}} \int_{\Omega} f_{1}(x)\left|u_{n}\right|^{r(x)} d x \\
-\int_{\partial \Omega} G\left(x, u_{n}\right) d \sigma_{x}-\frac{\min (L, 1)}{\mu}\left\|u_{n}\right\|^{p^{-}}-\frac{\lambda}{\mu} \int_{\Omega} f_{1}(x)\left|u_{n}\right|^{r(x)} d x-\frac{1}{\mu} \int_{\partial \Omega} g\left(x, u_{n}\right) u_{n} d \sigma_{x} \\
\geq \min (L, 1)\left(\frac{1}{p^{+}}-\frac{1}{\mu}\right)\left\|u_{n}\right\|^{p^{-}}-\left(\frac{\lambda}{r^{+}}+\frac{\lambda}{\mu}\right) \int_{\Omega} f_{1}(x)\left|u_{n}\right|^{r(x)} d x+\int_{\partial \Omega}\left(\frac{1}{\mu} g\left(x, u_{n}\right) u_{n}-G\left(x, u_{n}\right)\right) d \sigma_{x} \\
\geq \min (L, 1)\left(\frac{1}{p^{+}}-\frac{1}{\mu}\right)\left\|u_{n}\right\|^{p^{-}}-\left(\frac{\lambda}{r^{+}}+\frac{\lambda}{\mu}\right)\left|f_{1}\right|_{L^{\frac{p(x)}{p(x)-r(x)}(\Omega)}} C_{r^{+}}\left\|u_{n}\right\|^{r^{+}},
\end{gathered}
$$


where $d$ is defined in (3.1). Since $p^{-} \geq r^{+}\left(u_{n}\right)$ is bounded.

Now, with standard arguments, we prove that any $(P S)_{d}$ sequence $\left(u_{n}\right)$ in $X$ has a convergent subsequence. Indeed, the space $X$ is a Banach reflexive space then there exists $u \in X$ such that, up to subsequence still denoted by $\left(u_{n}\right)$ and by the Sobolev embedding, we obtain:

- $u_{n} \rightarrow u$ in $X \quad$ as $n \rightarrow \infty$;

- $u_{n}(x) \rightarrow u(x) \quad$ a.e. $\quad$ in $\Omega \quad$ as $n \rightarrow \infty$;

- $u_{n} \rightarrow u \quad$ in $L^{p(x)}(\Omega)$ as $n \rightarrow \infty$;

- $u_{n} \rightarrow u \quad$ in $\quad L^{\frac{p(x)}{p(x)-1}}(\Omega) \quad$ as $\quad n \rightarrow \infty$.

Proposition 8. If $u_{n} \rightarrow u$ in $X$ as $n \rightarrow \infty$, then

$$
\lim _{n \rightarrow \infty} \int_{\Omega} f_{1}(x)\left|u_{n}\right|^{r(x)-1}\left(u_{n}-u\right) d x=0
$$

and

$$
\lim _{n \rightarrow \infty} \int_{\partial \Omega} g_{1}(x)\left|u_{n}\right|^{q(x)-1}\left(u_{n}-u\right) d \sigma_{x}=0 .
$$

P r o o f. To demonstrate (3.6), we use Propositions 1-4 we give

$$
\begin{aligned}
& \int_{\Omega} f_{1}(x)\left|u_{n}\right|^{r(x)-1}\left(u_{n}-u\right) d x \leq \int_{\Omega}\left|f_{1}(x)\right|\left|u_{n}\right|^{r(x)-1}\left|u_{n}-u\right| d x \\
& \leq 3 C\left|f_{1}\right|_{L^{\frac{p(x)}{p(x)-r(x)}(\Omega)}} \max \left(\left|u_{n}\right|_{p(x)}^{r^{-}-1},\left|u_{n}\right|_{p(x)}^{r^{+}-1}\right)\left|u_{n}-u\right|_{p(x)},
\end{aligned}
$$

where $C$ is positive constant. By the compact embedding $X \hookrightarrow L^{p(x)}(\Omega)$ and the inequality $\left.|| u_{n}\right|_{p(x)}-|u|_{p(x)}|\leq| u_{n}-\left.u\right|_{p(x)}$, we obtain $\left|u_{n}-u\right|_{p(x)} \rightarrow 0$ in $L^{p(x)}(\Omega)$ and $\left|u_{n}\right|_{p(x)} \rightarrow|u|_{p(x)}$.

Similar arguments establish (3.7).

Now, in virtue of (3.1) and Proposition 8, we have

$$
\begin{aligned}
& \limsup _{n \rightarrow \infty} \int_{\Omega} a\left(\left|\nabla u_{n}\right|^{p(x)}\right)\left|\nabla u_{n}\right|^{p(x)-2} \nabla u_{n}\left(\nabla u_{n}-\nabla u\right) d x+\int_{\partial \Omega} b(x)\left|u_{n}\right|^{p(x)-2} u_{n}\left(u_{n}-u\right) d \sigma_{x} \\
= & \limsup _{n \rightarrow \infty} I_{\lambda}^{\prime}\left(u_{n}\right) \cdot u_{n}+\limsup _{n \rightarrow \infty} \lambda \int_{\Omega} f\left(x, u_{n}\right)\left(u_{n}-u\right) d x+\limsup _{n \rightarrow \infty} \int_{\partial \Omega} g\left(x, u_{n}\right)\left(u_{n}-u\right) d \sigma_{x}=0 .
\end{aligned}
$$

Finally, by Lemma $1 u_{n} \rightarrow u$ in $X$.

To finish the proof of the Theorem 1, we check the geometrical conditions of mountain pass Theorem 2 for $I_{\lambda}$. Indeed

$\left(\mathbf{I}_{1}\right)$ since the embeddings $X \hookrightarrow L^{i(x)}(\Omega)(i:=p, r, q)$ and $X \hookrightarrow L^{i(x)}(\partial \Omega)(i:=p, q)$ is are compact, there exist positive constants $C_{i}$ such that

$$
|u|_{i(x)} \leq C_{i}\|u\| .
$$

From $\left(\mathbf{G}_{0}\right)-\left(\mathbf{G}_{1}\right)$ it follows, for all $\varepsilon>0$, there exists $C_{\varepsilon}>0$, such that

$$
G(x, u) \leq \frac{\varepsilon}{p^{+}}|u|^{p^{+}}+C_{\varepsilon}|u|^{q(x)}, \quad \text { for all } \quad(x, t) \in \partial \Omega \times \mathbb{R},
$$


thus, for $u \in X$ with $\|u\| \leq 1$. By $\left(\mathbf{A}_{1}\right),(3.2),(3.4)$, (3.8) and (3.9), we have

$$
\begin{gathered}
I_{\lambda}(u) \geq \frac{\min (L, 1)}{p^{+}}\|u\|^{p^{+}}-\frac{\lambda C_{r}\left|f_{1}\right|_{L^{p(x) /(p(x)-r(x))}(\Omega)}}{r^{-}}\left\|u_{n}\right\|^{r^{+}}-\frac{\varepsilon C_{\varepsilon} C_{p}}{p^{+}}\|u\|^{p^{+}}-C_{q} C_{g}\|u\|^{q^{+}} \\
\geq\|u\|^{p^{+}}\left[C_{1}-\lambda C_{2}\|u\|^{r^{+}-p^{+}}-C_{3}\|u\|^{q^{+}-p^{+}}\right]
\end{gathered}
$$

where

$$
C_{1}=\frac{\min (L, 1)}{p^{+}}-\frac{\varepsilon C_{\varepsilon} C_{p}}{p^{+}}, \quad C_{2}=\frac{C_{r}\left|f_{1}\right|_{L^{p(x) /(p(x)-r(x))}(\Omega)}}{r^{-}}, \quad C_{3}=C_{q} C_{g} .
$$

If $\rho=\|u\|$, we obtain

$$
I_{\lambda}(u) \geq \rho^{p^{+}} \overbrace{\left[C_{1}-\lambda C_{2} \rho^{r^{+}-p^{+}}-C_{3} \rho^{q^{+}-p^{+}}\right]}^{\psi(\rho)} .
$$

A straightforward computation shows that the maximum of the function $\psi$ is

$$
\rho_{m}=\left(\frac{q^{+}\left(p^{+}-r^{+}\right) \lambda C_{2}}{r^{+}\left(q^{+}-r^{+}\right) C_{3}}\right)
$$

Inserting this into equation (3.11), we find that the right side is zero for

$$
\lambda^{*}:=\frac{C_{3}}{C_{2}} \rho_{m}^{q^{+}-r^{+}}-\frac{C_{1}}{C_{2}} \rho_{m}^{p^{+}-r^{+}} .
$$

So, there exist $\rho>0$ and $\varrho>0$ such that $I_{\lambda}(u) \geq \varrho$ for $\|u\|=\rho$, from which the demonstration of $\left(\mathbf{I}_{1}\right)$ is completed.

Now, put

$$
h(\tau)=\tau^{-\mu} G(x, \tau t)-G(x, t) \quad \forall t \geq 1 .
$$

We have

$$
h^{\prime}(t)=t^{-\mu-1}(g(x, t \tau) t \tau-G(x, t \tau)) \geq 0 \quad \forall t \geq 1
$$

by $\left(\mathbf{G}_{2}\right)$. Hence, $h(\tau) \geq h(1)$ for all $\tau \geq 1$ that is,

$$
G(x, \tau t) \geq \tau^{\mu} G(x, t) \quad \forall(x, t) \in \partial \Omega \times \mathbb{R} .
$$

Let $u \in X$, for $t>1$, by $\left(\mathbf{A}_{0}\right)$ and (3.12), we have

$$
\begin{gathered}
I_{\lambda}(t u)=\int_{\Omega} \frac{1}{p(x)} A\left(|\nabla t u|^{p(x)}\right) d x+\int_{\partial \Omega} \frac{1}{p(x)} b(x)|t u|^{p(x)} d \sigma_{x}-\lambda \int_{\Omega} F(x, t u) d x-\int_{\partial \Omega} G(x, t u) d \sigma_{x} \\
\leq t^{p^{+}}\left(\int_{\Omega} \frac{1}{p(x)} A\left(|\nabla u|^{p(x)}\right) d x+\int_{\partial \Omega} \frac{1}{p(x)} b(x)|u|^{p(x)} d \sigma_{x}\right) \\
+t^{r^{+}} \frac{\lambda}{r^{+}} \int_{\Omega} f_{1}(x)|u|^{r(x)} d x-C_{4} t^{\mu} \int_{\partial \Omega}\left[\frac{\varepsilon}{p^{+}}|u|^{p^{+}}+C_{\varepsilon}|u|^{q(x)}\right] d \sigma_{x} .
\end{gathered}
$$

This shows that $I_{\lambda}(t u)<0$.

Since $I_{\lambda}(0)=0$, the mountain pass lemma implies the existence of a nontrivial weak solution $u_{1}$ with $I_{\lambda}\left(u_{1}\right) \geq \varrho$.

Hence problem (1.1) has at least one nontrivial weak solution in $X$.

To complete the proof of the Theorem 1, one must check the conditions of the Theorem 3. So we need some lemmas which we recall below. 
Remark 2. [30] As the Sobolev space $X$ is a reflexive and separable Banach space, there exist $\left(e_{n}\right)_{n \in \mathbb{N}^{*}} \subseteq X$ and $\left(f_{n}\right)_{n \in \mathbb{N}^{*}} \subseteq X^{*}$ such that $f_{n}\left(e_{m}\right)=\delta_{n m}$ for any $n, m \in \mathbb{N}^{*}$ and

$$
X=\overline{\operatorname{span}\left\{e_{n}: n \in \mathbb{N}^{*}\right\}}, \quad X^{*}=\overline{\operatorname{span}\left\{f_{n}: n \in \mathbb{N}^{*}\right\}} w^{*} .
$$

For $k \in \mathbb{N}^{*}$ denote by $X_{k}=\operatorname{span}\left\{e_{k}\right\}, \quad Y_{k}=\oplus_{j=1}^{k} X_{j}, \quad Z_{k}=\overline{\oplus_{k}^{\infty} X_{j}}$.

Lemma 3. Assume that $\left(\mathbf{A}_{0}\right)-\left(\mathbf{A}_{1}\right),\left(\mathbf{F}_{0}\right)-\left(\mathbf{F}_{1}\right)$ and $\left(G_{0}\right)-\left(G_{1}\right)$ hold. Then there exists $\tilde{\lambda}>0$, $k \in \mathbb{N}$ and $\rho, \theta>0$ such that $I_{\lambda} / \partial B_{\rho} \cap X_{k} \geq \theta$ for all $0<\lambda<\widetilde{\lambda}$.

P r o o f. Similarly to (3.10), we have

$$
I_{\lambda}(u) \geq\|u\|^{p^{+}}\left[C_{1}-\lambda C_{2}\|u\|^{r^{+}-p^{+}}\right]-C_{3}\|u\|^{q^{+}} .
$$

Taking $\rho=\|u\|$, we get

$$
I_{\lambda}(u) \geq \rho^{p^{+}}\left[C_{1}-\lambda C_{2} \rho^{r^{+}-p^{+}}\right]-C_{3} \rho^{q^{+}} .
$$

Next, we take $\tilde{\lambda}=C_{1} / C_{2} \cdot \rho^{p^{+}-r^{+}}>0$ so that

$$
I_{\lambda}(u) \geq \rho^{p^{+}}\left[C_{1}-\lambda C_{2} \rho^{r^{+}-p^{+}}\right]-C_{3} \rho^{q^{+}}>0,
$$

which shows that $I$ verifies the condition $\left(\mathbf{I}_{1}\right)$ in Theorem 3.

Finally, to show the condition $\left(\mathbf{I}_{2}{ }_{2}\right)$ in Theorem 3, we use the following lemma.

Lemma 4. Assume that $\left(\mathbf{A}_{0}\right)-\left(\mathbf{A}_{1}\right)$ and $\left(\mathbf{G}_{2}\right)-\left(\mathbf{G}_{3}\right)$ hold. Then, given $m \in \mathbb{N}$, there exist a subspace $W$ of $X$ and a constant $M_{m}>0$, independent of $\lambda$, such that $\operatorname{dim} W=m$ and $\max _{u \in W} I_{\lambda}(u)<M_{m}$.

$\mathrm{P}$ r o o f. Let $O$ and $U$ be defined respectively as in $\left(\mathbf{F}_{1}\right)$ and in $\left(\mathbf{G}_{3}\right)$. We can build the space $W$, in the same way as in [28, Lemma 4.3]. So, we consider $v_{1}, \ldots \ldots, v_{m}$ such that $v_{i} \in C_{O}^{\infty}(\Omega)$, $\operatorname{supp} v_{i} \cap \operatorname{supp} v_{j}=\emptyset, \operatorname{supp} v_{i} \cap O \neq \emptyset$ and $\operatorname{supp} v_{i} \cap U \neq \emptyset$, where $i=1, \ldots, m, j=1, \ldots, m, i \neq j$.

By (2), we have

$$
\begin{gathered}
I_{\lambda}(u)=\int_{\Omega} \frac{1}{p(x)} A\left(|\nabla u|^{p(x)}\right) d x+\int_{\partial \Omega} \frac{1}{p(x)} b(x)|u|^{p(x)} d \sigma_{x}-\lambda \int_{\Omega} F(x, u) d x-\int_{\partial \Omega} G(x, u) d \sigma_{x} \\
\leq \frac{\max (1, K)}{p^{-}} \max \left(\|u\|^{p^{-}},\|u\|^{p^{+}}\right)-\lambda \int_{\Omega} F(x, u) d x-\int_{\partial \Omega} G(x, u) d \sigma_{x},
\end{gathered}
$$

where $K$ is defined in $\left(\mathbf{A}_{0}\right)$.

For $u \in W$, since supp $u \cap O \neq \emptyset$ we get

$$
I_{\lambda}(u) \leq \frac{\max (1, K)}{p^{-}} \max \left(\|u\|^{p^{-}},\|u\|^{p^{+}}\right)-\int_{\partial \Omega} G(x, u) d \sigma_{x}=\widetilde{I}(u) .
$$

Since

$$
\max _{u \in W \backslash\{0\}} I_{\lambda}(u) \leq \max _{u \in W \backslash\{0\}} \widetilde{I}(u)=\max _{v \in \partial B_{1}(0) \cap W \backslash\{0\}} \widetilde{I}(v) .
$$

For $t>0$ and $u \in \partial B_{1}(0) \cap W \backslash\{0\}$ and $\varepsilon$ small enough, by $\left(\mathbf{F}_{1}\right),\left(\mathbf{G}_{2}\right)-\left(\mathbf{G}_{3}\right)$ and (3.9), we obtain

$$
\begin{gathered}
\widetilde{I}(t u)=\frac{\max (1, K)}{p^{-}} \max \left(\|t u\|^{p^{-}},\|t u\|^{p^{+}}\right)-\int_{\partial \Omega} G(x, t u) d \sigma_{x} \\
\leq C_{5}\|t u\|^{p^{-}}-t^{\mu} \int_{\partial \Omega}\left(\frac{\varepsilon}{p^{+}}|u|^{p^{+}}+C_{\varepsilon}|u|^{q(x)}\right) d \sigma_{x} \leq C_{5} t^{p^{-}}\|u\|^{p^{-}}-C_{6} t^{\mu}\|u\|^{q^{-}},
\end{gathered}
$$


where $C_{5}=\max (1, K) / p^{-}$and $C_{6}$ is the constant of embedding $X \hookrightarrow L^{q(x)}(\partial \Omega)$,

$$
\lim _{t \rightarrow+\infty} \widetilde{I}(t u) \leq \lim _{t \rightarrow+\infty}\left[C_{5} t^{p^{-}}-C_{6} t^{\mu}\right] .
$$

Since $\mu>p^{-}$, by (3.13) we get that there exist a subspace $W$ of $X$ and a constant $M_{m}>0$, independent of $\lambda$, such that $\operatorname{dim} W=m$ and $\max _{u \in W} I_{\lambda}(u)<M_{m}$. The proof of Lemma 4 is complete. $\square$

According to Lemma 2, we also have that $I_{\lambda}$ satisfies $\left(\mathbf{I}_{3}\right)$. Since $I_{\lambda}(0)=0$ and $I_{\lambda}$ is even, we may apply Theorem 3 to conclude that $I_{\lambda}$ has infinitely many nontrivial solutions.

\section{Acknowledgements}

The authors would like to thank the referees for their valuable comments which improved the presentation of the original manuscript.

\section{REFERENCES}

1. Allaoui M., El Amrouss A., Ourraoui A. Existence of infinitely many solutions for a Steklov problem involving the $p(x)$-Laplace operator. Electron. J. Qual. Theory Differ. Equ., 2014. No. 20. P. 1-10. DOI: 10.14232/ejqtde.2014.1.20

2. Antontsev S., Shmarev S., Chapter 1. Elliptic equations with anisotropic nonlinearity and nonstandard growth conditions. In: Handbook of Differential Equations, Stationary Partial Differ. Equ. Chipot M., Quittner P. (eds.), 2006. Vol. 3. P. 1-100. DOI: 10.1016/S1874-5733(06)80005-7

3. Bocea M., Mihăilescu M. Г-convergence of power-law functionals with variable exponents. Nonlinear Anal.: Theory, Methods, Appl., 2010. Vol. 73, No. 1. P. 110-121. DOI: 10.1016/j.na.2010.03.004

4. Bocea M., Mihăilescu M., Popovici C. On the asymptotic behavior of variable exponent power-law functionals and applications. Ric. Mat., 2010. Vol. 59. P. 207-238. DOI: 10.1007/s11587-010-0081-x

5. Chabrowski J., Fu Y. Existence of solutions for $p(x)$-Laplacian problems on a bounded domain. J. Math. Anal. Appl., 2005. Vol. 306, No. 2. P. 604-618. DOI: 10.1016/j.jmaa.2004.10.028

6. Chen Y., Levine S., Rao M. Variable exponent, linear growth functionals in image restoration. SIAM J. Appl. Math., 2006. Vol. 66, No. 2. P. 1383-1406. DOI: 10.1137/050624522

7. Dai G. Infinitely many solutions for a $p(x)$-Laplacian equation in $\mathbb{R}^{N}$. Nonlinear Anal.: Theory, Methods, Appl., 2009. Vol. 71, No. 3-4. P. 1133-1139. DOI: 10.1016/j.na.2008.11.037

8. Deng S.-G. Positive solutions for Robin problem involving the $p(x)$-Laplacian. J. Math. Anal. Appl., 2009 Vol. 360, No. 2. P. 548-560. DOI: 10.1016/j.jmaa.2009.06.032

9. Deng S.-G. Eigenvalues of the $p(x)$-Laplacian Steklov problem. J. Math. Anal. Appl., 2008. Vol. 339, No. 2. P. 925-937. DOI: 10.1016/j.jmaa.2007.07.028

10. Deng S.-G. A local mountain pass theorem and applications to a double perturbed $p(x)$-Laplacian equations. Appl. Math. Comput., 2009. Vol. 211, No. 1. P. 234-241. DOI: 10.1016/j.amc.2009.01.042

11. Diening L., Hästö P., Nekvinda A. Open problems in variable exponent Lebesgue and Sobolev spaces. In: Function Spaces, Differential Operators And Nonlinear Analysis. Proc. Conference Held in Milovy, Bohemian-Moravian Uplands. Drábek P., Rákosnik J. (Eds.) May 28 - June 2, 2004, Milovy, Czech Republic. Milovy: Math. Inst. Acad. Sci. Czech, 2005. P. 38-58. URL: https://citeseerx.ist.psu.edu

12. Diening L., Harjulehto P., Hästö P., Ruzicka M. Lebesgue and Sobolev Spaces with Variable Exponents. Lecture Notes in Math., vol. 2017. Berlin, Heidelberg: Springer-Verlag, 2011. 509 p. DOI: $10.1007 / 978-3-642-18363-8$

13. Edmunds D. E., Rákosník J. Sobolev embeddings with variable exponent. Studia Math., 2000. Vol. 143, No. 3. P. 267-293.

14. El Amrouss A., Moradi F., Ourraoui A. Neumann problem in divergence form modeled on the $p(x)$-Laplace equation. Bol. Soc. Parana. Mat. (3), 2014. Vol. 32, No. 2. P. 109-117. DOI: $10.5269 /$ bspm.v32i2.20329

15. Fan X., Han X. Existence and multiplicity of solutions for $p(x)$-Laplacian equations in $\mathbb{R}^{N}$. Nonlinear Anal.: Theory Methods Appl., 2004. Vol. 59. P. 173-188. DOI: 10.1016/j.na.2004.07.009 
16. Fan X., Zhao D. On the spaces $L^{p(x)}(\Omega)$ and $W^{m, p(x)}(\Omega)$. J. Math. Anal. Appl., 2001. Vol. 263, No. 2. P. 424-446. DOI: $10.1006 /$ jmaa.2000.7617

17. Fan X.-L., Zhang Q.-H. Existence of solutions for $p(x)$-Laplacian Dirichlet problem. Nonlinear Anal.: Theory Methods Appl., 2003. Vol. 52, No. 8. P. 1843-1852. DOI: 10.1016/S0362-546X(02)00150-5

18. Fu Y., Zhang X. A multiplicity result for $p(x)$-Laplacian problem in $\mathbb{R}^{N}$. Nonlinear Anal.: Theory, Methods, Appl., 2009. Vol. 70, No. 6. P. 2261-2269. DOI: 10.1016/j.na.2008.03.038

19. Juárez Hurtado E., Miyagaki O. H., Rodrigues R. S. Existence and Multiplicity of Solutions for a Class of Elliptic Equations Without Ambrosetti-Rabinowitz Type Conditions. J. Dynam. Differential Equations, 2018. Vol. 30. P. 405-432. DOI: 10.1007/s10884-016-9542-6

20. Kováčik O., Rákosník J. On spaces $L^{p(x)}$ and $W^{k, p(x)}$. Czechoslovak Math. J., 1991. Vol. 41, No. 4. P. 592-618. URL: https://dml.cz/handle/10338.dmlcz/102493

21. Mihăilescu M. On a class of nonlinear problems involving a $p(x)$-Laplace type operator. Czechoslovak Math. J., 2008. Vol. 58, No. 1. P. 155-172. URL: https://dml.cz/handle/10338.dmlcz/128252

22. Ni W.-M., Serrin J. Existence and non-existence theorems for ground states for quasilinear partial differential equations. Att. Conveg. Lincei, 1985. Vol. 7. P. 231-257.

23. Ourraoui A. Multiplicity results for Steklov problem with variable exponent. Appl. Math. Comput., 2016. Vol. 277. P. 34-43. DOI: 10.1016/j.amc.2015.12.043

24. Ourraoui A. Some Results for Robin Type Problem Involving $p(x)$-Laplacian. Preprint.

25. Pflüger K. Existence and multiplicity of solutions to a $p$-Laplacian equation with nonlinear boundary condition. Electron. J. Differential Equations, 1998. Vol. 1998, No. 10. P. 1-13. http://ejde.math.unt.edu

26. Rabinowitz P.H. Minimax Methods in Critical Point Theory with Applications to Differential Equations. CBMS Regional Conference Series in Mathematics, Vol. 65. Providence, Rhode Island: American Mathematical Soceity, 1986. 100 p.

27. Růžička M. Electrorheological Fluids: Modeling and Mathematical Theory. Lecture Notes in Math., vol. 1748. Berlin, Heidelberg: Springer-Verlag, 2002. 178 p. DOI: 10.1007/BFb0104029

28. Silva E.A.B., Xavier M.S. Multiplicity of solutions for quasilinear elliptic problems involving critical Sobolev exponents. Ann. Inst. H. Poincaré Anal. Non Linéaire, 2003. Vol. 20, No. 2. P. 341-358. DOI: $10.1016 / \mathrm{S} 0294-1449(02) 00013-6$

29. Zhao D., Fan X. L. On the Nemytskiĩ operators from $L^{p_{1}(x)}(\Omega)$ to $L^{p_{2}(x)}(\Omega)$. J. Lanzhou Univ. Nat. Sci., 1998. Vol. 34, No. 1. P. 1-5. (in Chinese)

30. Zhao J. F. Structure Theory of Banach Spaces. Wuhan: Wuhan Univ. Press, 1991. (in Chinese)

31. Zhou Q.-M., Ge B. Multiple solutions for a Robin-type differential inclusion problem involving the p(x)-Laplacian. Math. Methods Appl. Sci., 2013. Vol. 40, No. 18. P. 6229-6238. DOI: 10.1002/mma.2760

32. Zhikov V. V. Averaging of functionals of the calculus of variations and elasticity theory. Math. USSR-Izv., 1987. Vol. 29, No. 1. P. 33-66. DOI: 10.1070/IM1987v029n01ABEH000958 